\section{A COMPARISON OF ELECTROGRAPHIC SEIZURE CHARACTERISTICS IN TERM NEONATES WITH HYPOXIC-ISCHAEMIC ENCEPHALOPATHY AND FOCAL CEREBRAL LESIONS}

\author{
E. Low, B. Walsh, C. Ryan, G. Boylan \\ Neonatal Brain Research Group, University \\ College Cork, Cork, Ireland
}

Background and aims: In term neonates with seizures, it is important to distinguish between hypoxic-ischaemic encephalopathy (HIE) and focal cerebral lesions as soon as possible to aid clinical intervention. We compared seizure onset, duration and morphology in term neonates with HIE and focal cerebral lesions.

Methods: Term neonates with perinatal distress or abnormal neurology were recruited. Continuous multichannel video-EEG recording within 72 hours of birth was performed.

Results: In 26 term neonates recruited, 13 had EEG seizures $[\operatorname{HIE}(n=5)$; focal arterial infarction $(n=3)$; focal intraparenchymal haemorrhage $(n=2)$; unknown pathology $(n=3)]$. EEG recordings commenced at a median age(range) of $6.0(1.8-59.2)$ hours. The median recording duration was $61.4(24.0-122.1)$ hours.

The median seizure onset occurred earlier in HIE than in focal cerebral lesions [13.4(8.3-42.2) hours vs 38.6(18.0-59.7) hours]. The median total seizure burden per neonate with HIE was higher than in focal cerebral lesions [212.4(55.2-640.2) minutes vs 112.9(12.9-189.1) minutes]. Seizures were more frequent in HIE than in focal cerebral lesions (577 vs 113 events). Two neonates with severe HIE also had status epilepticus.

Electrographic seizures in neonates with focal cerebral lesions originated from the site of injury displayed a characteristic spike wave focal morphology with minimal frequency change. Variable seizure morphologies and frequencies were seen in HIE. The background EEG activity in focal cerebral lesions was well preserved, though asymmetries were evident. The background EEG activity in HIE showed global abnormalities.

Conclusions: The contrastingseizurecharacteristics in neonates with HIE and focal cerebral lesions may prove useful for automated seizure detection systems and guide appropriate management strategies.

\section{FOLLOW-UP OF NEONATAL NON- HAEMOLYTIC HYPERBILIRUBINEMIA IN DANISH TERM AND NEAR-TERM INFANTS WITH TOTAL SERUM BILIRUBIN LEVEL $\geq 420$ UMOL/L}

\author{
P.K. Vandborg ${ }^{1}$, G. Greisen², B.M. Hansen², \\ F. Ebbesen ${ }^{1}$ \\ ${ }^{1}$ Department of Pediatrics, Aalborg University \\ Hospital, Aalborg, 'Department of Neonatology, \\ Copenhagen University Hospital, Copenhagen, \\ Denmark
}

Background: It is well described that infants with severe non-haemolytic hyperbilirubinemia in the neonatal period, who present with acute encephalopathy may suffer from permanent brain damage, kernicterus. Whether the infants with severe hyperbilirubinemia presenting with minor or no neurological symptoms in the neonatal period may sustain more subtle brain injury resulting in developmental delay remain controversial.

Objective: To study if severe non-haemolytic hyperbilirubinemia in infants with minor or no neurological symptoms in the neonatal period affects the childrens development at the age of 1-5 years.

Design/methods: A controlled descriptive study evaluating all infants born in Denmark 2004-2007 with a gestational age 35 weeks and severe neonatal non-haemolytic hyperbilirubinemia with total serum bilirubin (TSB) $\geq 420$ umol/l. Total births those years were approximately 240.000 . The study group of 211 (0.9 per 1000) children was identified by linking electronically stored laboratory data with the Danish personal identification number and next medical records of all children with TSB $\geq 420$ umol/l was found. A control group of 208 children matched to the study group on sex, age, GA and municipality of residence was found through the Danish birth registry. The families to the children were mailed the Ages and Stages Questionnaire (ASQ), which is a parent completed questionnaire evaluating the childs development.

Results: The response rate was $79 \%$ for the study group and $70 \%$ for the control group. There were no differences in ASQ score between the groups.

Conclusions: We found no evidence of developmental delay in children with severe neonatal non-haemolytic hyperbilirubinemia. 